\title{
Successful transcatheter aortic valve implantation (TAVI) is associated with transient left ventricular dysfunction
}

\author{
Rafal Dworakowski, Olaf Wendler, Amit Bhan, Lindsay Smith, Peter Pearson, \\ Emma Alcock, Kailasam Rajagopal, Beth Brickham, Tracy Dew, Jonathan Byrne, \\ Mark J Monaghan, Roy Sherwood, Ajay M Shah, Philip A MacCarthy
}

\begin{abstract}
King's College London BHF Centre of Excellence, Department of Cardiology, King's College Hospital, London, UK
\end{abstract}

\section{Correspondence to} Dr Philip MacCarthy, Consultant Cardiologist, Department of Cardiology, King's College Hospital, Denmark Hill, London SE5 9RS, UK; philip. maccarthy@nhs.net

Accepted 27 July 2012

Published Online First 21 August 2012

\begin{abstract}
Objective To investigate early haemodynamic changes after transfemoral transcatheter aortic valve implantation (TAVI) and the relationship with myocardial injury and neurohormonal activation.

Design Single-centre prospective observational study. Setting Tertiary cardiac centre.

Patients 42 patients undergoing transfemoral TAVI were included in this study.

Main outcome measures Haemodynamic measurements and echocardiography-derived indices characterising myocardial function were recorded at baseline, 6 and $24 \mathrm{~h}$ postprocedure. Postprocedural myocardial injury was quantified using serum troponin I and CK-MB levels. In addition, biomarkers of myocardial dysfunction/heart failure and neurohormonal activation were measured.
\end{abstract}

Results $6 \mathrm{~h}$ Post-TAVI there was a significant deterioration in both systolic and diastolic function as measured by $\mathrm{dP} / \mathrm{dt}_{\max } / \mathrm{EDV}$, myocardial performance index and mean $\mathrm{E} / \mathrm{e}^{\prime}$ index. Recovery of myocardial function was observed at $24 \mathrm{~h}$. These haemodynamic changes were associated with a significant increase in both troponin I $(0.07 \pm 0.01$ vs $1.59 \pm 0.21 \mu \mathrm{g} / \mathrm{l}$, $\mathrm{p}<0.005)$ and CK-MB $(1.99 \pm 0.19$ vs $6.82 \pm 0.7 \mathrm{ng} / \mathrm{ml}$, $p<0.005)$. There was a positive correlation among myocardial injury and NT-BNP $(r=0.34, p<0.0005)$, aldosterone $(r=0.56, p<0.0001)$ and ST2 levels $(r=0.21, p<0.05)$.

Conclusions This is the first study to demonstrate that procedurally successful TF-TAVI results in a transient depression of both systolic and diastolic left ventricular function within the first 24 postoperative hours, despite impressive relief of previously severe, chronic pressure overload. The rise in the markers of myocardial damage suggests that this may be due to periprocedural myocardial injury. Complete recovery of contractility is generally observed after $24 \mathrm{~h}$.

\section{INTRODUCTION}

Chronic pressure overload induced by a stenotic aortic valve results in concentric left ventricular (LV) hypertrophy and diastolic dysfunction and, thereafter, LV systolic decompensation and contractile dysfunction. Surgical aortic valve replacement (sAVR) is known to result in transient perioperative deterioration of LV contractile function, with diastolic LV dysfunction thought to be related to the effects of cardiopulmonary bypass. ${ }^{1}$ In addition to these perioperative haemodynamic changes, increased neurohormonal activation and myocardial injury following conventional cardiac surgery correlate with perioperative complications and predict development of heart failure and mortality in the longer-term. ${ }^{2}$

Transcatheter aortic valve implantation (TAVI) is a novel procedure for the treatment of severe aortic stenosis without the need for cardiopulmonary bypass. TAVI can be performed purely endovascularly (without thoracotomy) using a transfemoral (TF) approach. The procedure results in almost instantaneous normalisation of transvalvular gradients with abrupt release of chronic pressure overload, but little is yet known about the periprocedural haemodynamic changes, myocardial injury and neurohormonal activation accompanying this process.

The aim of this study was to define the immediate haemodynamic changes occurring after TF-TAVI. We utilised transthoracic echocardiography and continuous cardiac output (CO) monitoring and also assessed markers of myocardial damage (troponin I and (CK-MB) Creatine Kinase $M B$ fraction) and neurohormonal activation (NT-pro-BNP, ST2 and aldosterone).

\section{METHODS \\ Patients}

We prospectively studied 42 consecutive patients with severe aortic stenosis who were referred to King's College Hospital for TAVI and had a TF procedure (TF-TAVI) using the Edwards-Sapien XT (Edwards Life- sciences, Inc, California, USA) valve between May 2009 and December 2011. The study was approved by the local research ethics committee and all subjects provided written informed consent. The patient characteristics are summarised in table 1 . In all, $66 \%(n=28)$ of patients had at least one coronary stenosis $>50 \%$, with $38 \%(n=16)$ who had single vessel disease, $23 \% \quad(n=12)$ who had severe ischaemic heart disease (three vessel disease or proximal LAD/LM main disease) but only $19 \%$ patients who had angina before TAVI.

\section{TAVI procedure}

Transfemoral TAVI was performed using the conventional technique which has been described elsewhere. ${ }^{3}$ General anaesthesia was used in all 
Table 1 Clinical characteristics of patient cohort

\begin{tabular}{|c|c|}
\hline & $\mathrm{N}=42$ \\
\hline \multicolumn{2}{|l|}{ Baseline clinical characteristics } \\
\hline Age (years $\pm S D$ ) & $83 \pm 6$ \\
\hline Female gender $\mathrm{n}(\%)$ & $25(59 \%)$ \\
\hline Weight $(\mathrm{kg}$, mean $\pm S D)$ & $70 \pm 19$ \\
\hline logistic EuroSCORE (mean $\pm S D)$ & $22 \pm 11$ \\
\hline Diabetes $(\%)$ & $6(14 \%)$ \\
\hline Hypertension (\%) & $32(76 \%)$ \\
\hline Carotid artery stenosis $>50 \%(\%)$ & $9(21 \%)$ \\
\hline Peripheral vascular disease $(\%)$ & $3(7 \%)$ \\
\hline LV function $<30 \%$ & $3(7 \%)$ \\
\hline Creatine (umol/l) & $93.6 \pm 31.8$ \\
\hline eGFR (ml/min) & $61.4 \pm 16.2$ \\
\hline $\begin{array}{l}\text { Coronary artery disease (>50\% } \\
\text { one and more vessel) (\%) }\end{array}$ & $28(66 \%)$ \\
\hline $\begin{array}{l}\text { Severe coronary artery disease (stenosis }>50 \% \text { in } \\
\text { two or more vessels or LM disease) }\end{array}$ & $12(28 \%)$ \\
\hline Angina $(\%)$ & $8(19 \%)$ \\
\hline Previous MI (\%) & $8(19 \%)$ \\
\hline Prior coronary angioplasty & $9(21 \%)$ \\
\hline Previous cardiac surgery $(\%)$ & $7(17 \%)$ \\
\hline NYHA class III/IV (\%) & $34(81 \%)$ \\
\hline \multicolumn{2}{|l|}{ Baseline echocardiographic characteristics } \\
\hline Aortic valve area $\left(\mathrm{cm}^{2}\right)$ & $0.67 \pm 0.19$ \\
\hline Mean pressure gradient $(\mathrm{mm} \mathrm{Hg})$ & $47 \pm 19$ \\
\hline Peak pressure gradient $(\mathrm{mm} \mathrm{Hg})$ & $84 \pm 29$ \\
\hline Ejection fraction (\%) & $51 \pm 13$ \\
\hline Mitral regurgitation $\geq$ moderate $(\%)$ & $3(7 \%)$ \\
\hline
\end{tabular}

LV, left ventricular; MI, myocardial infarction; eGFR, estimated glomerular filtration rate; LM, Left main; NYHA, New York Heart Association.

cases and surgical exposure of the femoral artery was used as the preferred mode of arterial access. Balloon aortic valvuloplasty was performed prior to Sapien deployment in all cases and the device was deployed using the NovaFlex (Edwards LifeSciences, Irvine, California, US) delivery catheter, under conditions of rapid pacing $(180-200 \mathrm{bpm})$. Total time of rapid pacing time was recorded at the end of procedure. A similar anaesthetic technique was used for all patients undergoing TAVI. Anaesthesia was induced with a combination of midazolam (1-2 mg), fentanyl $(0.5-1 \mathrm{mcg} / \mathrm{kg})$ and propofol $(0.5-2 \mathrm{mg} / \mathrm{kg})$. Tracheal intubation was facilitated with vecuronium $(0.1 \mathrm{mg} / \mathrm{kg})$. Anaesthesia was maintained with sevoflurane in a mixture of oxygen and air, and remifentanil (up to $0.1 \mathrm{mcg} / \mathrm{kg} / \mathrm{min}$ ) infusion intraoperatively. Postoperative analgesia consisted of local anaesthetic infiltration, regular paracetamol, codeine and intravenous morphine as required. Initial fluid replacement was with compound sodium lactate $1000 \mathrm{ml}$. Blood pressure was maintained (systolic $>100 \mathrm{~mm} \mathrm{Hg}$ ) during the procedure with metaraminol boluses $(50-100 \mathrm{mcg})$. None of the patients required catecholamine (or other inotrope) infusion after the procedure.

TAVI outcome and complications were recorded using VARC definitions. ${ }^{4}$

\section{Invasive CO monitoring (Vigileo/FloTrac system)}

In each patient, a radial arterial cannula was inserted preoperatively and maintained during the immediate postoperative period. The FloTrac system (consisting of the Vigileo monitor and sensor) uses a clinically validated algorithm to provide continuous $\mathrm{CO}$, stroke volume and systemic vascular resistance in realtime. The Vigileo (Edwards Lifesciences) monitor with software V.1.01 was connected to the radial artery catheter via the FloTrac (Edwards Lifesciences) pressure sensor. This system calculates continuous $\mathrm{CO}$ on arterial pressure waveform characteristics.
Individual demographic data, including height, weight, age, gender and the real-time arterial pressure waveform analysis, are used to estimate arterial compliance.

\section{Echocardiography}

Transthoracic echocardiography was performed at three time points: (1) baseline (the day before the procedure); (2) the day of the procedure, $6 \mathrm{~h}$ after valve deployment; and (3) the day after the procedure at $24 \mathrm{~h}$.

At each time point a standard full adult echocardiogram, including 2D, pulsed and continuous Doppler, was performed. In addition, tissue Doppler of the mitral annulus was recorded and real time 3D datasets of the LV and LA cavities were acquired separately. Careful attention was paid to all acquisition settings in order to maximise image quality. Images were stored digitally and subsequently analysed blindly offline by $\mathrm{AB}$ and LS. All scans were performed using an iE33 ultrasound system (Philips Medical Systems, Bothell, Washington, USA) equipped with s5-1 (2D) and x3-1 (3D-matrix array) transducers and image analysis was performed using V.7 of Olab (Philips Medical Systems, Andover, Massachusetts, USA).

LV volumes were calculated using the $3 \mathrm{D}$ datasets (Olab Advanced) and LV ejection fraction was derived from this analysis. In addition, the preoperative images were used to obtain a 3D-derived estimation of LV mass. End-systolic LA volume was calculated from the 3D LA dataset. All 3D measurements were performed using previously well-described techniques. ${ }^{5}$

Medial and lateral as well as mean E:e' were calculated as estimates of LV filling pressures and systolic function/contractility was measured with $\mathrm{dP} / \mathrm{dt}$ max (calculated from the mitral regurgitation Doppler trace) and the early peak tissue Doppler systolic velocity $\left(\mathrm{S}^{\prime}\right)$. As an alteration in ventricular preload is known to affect $\mathrm{dP} / \mathrm{dt}_{\max }$, we normalised $\mathrm{dP} / \mathrm{dt}_{\max }$ for preload, which has been validated in previous studies. ${ }^{6}$ In addition, the Doppler-derived myocardial performance index (MPI, also denoted the Tei index, an index of combined systolic and diastolic function) was calculated. MPI is defined as the sum of isovolumic contraction time and isovolumic relaxation time divided by the ejection time. ${ }^{7}$

\section{Biomarkers}

Blood samples were obtained before TAVI and 6 and $24 \mathrm{~h}$ (at the same time points as echocardiography) after the procedure from a central line. Serum was isolated within $1 \mathrm{~h}$ of collection and samples were stored at $-80^{\circ} \mathrm{C}$ until thawed for determination of biomarkers.

\section{Markers of myocardial injury}

Serum CK-MB was measured using the Immulite 2000 assay (a chemiluminescent enzyme-labelled immunometric assay, reagent from Siemens Healthcare Diagnostics, UK). The imprecision of the assays is expressed as between-run coefficient of variation (\%CV). The between-run $\mathrm{CV}$ of the $\mathrm{CK}-\mathrm{MB}$ assay for concentrations of $13.9,54.3$ and $95.4 \mathrm{ng} / \mathrm{ml}$ was $5.8 \%, 5.5 \%$ and $6.1 \%$, respectively.

TropI was measured using the ADVIA Centaur TnI-Ultra assay (a three-site sandwich immunoassay using direct chemiluminometric technology, reagent from Siemens Healthcare Diagnostics). The functional sensitivity (the lowest TnI concentration determined at a CV of $20 \%$ ) is $0.017 \mu \mathrm{g} / \mathrm{l}$.

Markers of myocardial dysfunction and neurohormonal activation NT-pro-BNP was measured on the Siemens Immulite 2000 analyser (a two-site chemiluminescence immunoassay). The 
between-run CV of the BNP assay for concentrations of 35.6, 1430 and $29725 \mathrm{pg} / \mathrm{ml}$ was $5.4 \%, 3 \%$ and $4.1 \%$, respectively.

ST2 (R\&D Systems Europe, UK) was measured with a quantitative sandwich enzyme immunoassay technique. The between-run CV of the ST2 assay for concentrations of 273, 628 and $1027 \mathrm{pg} / \mathrm{ml}$ was $5.6 \%, 4.4 \%$ and $4.5 \%$, respectively.

Aldosterone (Siemens Medical Solutions Diagnostics, Los Angeles, California, USA) was measured with a solid-phase radioimmunoassay, based on aldosterone-specific antibody immobilised to the wall of a polypropylene tube. The betweenrun $\mathrm{CV}$ of the aldosterone assay for concentrations of 65,448 and $813 \mathrm{pg} / \mathrm{ml}$ was $3.5 \%, 15.3 \%$ and $19 \%$, respectively.

\section{STATISTICS}

Analyses were performed using the SPSS V.19 statistical package (SPSS Inc). Data were expressed as mean \pm SEM or percentage for categorical variables. Comparisons between results before and after TAVI were performed using paired Student $t$ test for two time points, one-way analysis of variance followed by a Newman-Keuls test or a non-parametric Friedman followed by a Dunn test as appropriate for more than two time points. Fit to normal distribution was tested using Shapiro-Wilk test. Pearson correlation was used to assess association between two continues variables. $p$ Value $<0.05$ was considered to be statistically significant. Sample size was calculated based on pilot study consisting of first 10 patients.

\section{RESULTS}

\section{Procedure outcome}

The valve prosthesis was successfully deployed in $100 \%$ of cases. There were no cases of more than mild aortic regurgitation. There was no periprocedural coronary occlusion, no clinically overt myocardial infarction nor stroke/TIA. Total pacing time was $45 \pm 4 \mathrm{~s}$ per patient. Inhospital and 30-day mortality were retrospectively $5 \%(\mathrm{n}=2 ; 81$-year-old male, died day 20 post-TAVI, cause of death: aspiration pneumonia, sepsis, multiorgan failure (Brain natriuretic peptide (BNP) 407 baseline vs $677324 \mathrm{~h}$ ); 87year-old male, died day 24 post-TAVI, cause of death: recurrent pulmonary oedema, multiorgan failure with renal impairment (BNP 1691 baseline vs 2678 24 h)) and 7\% (one further patient: 86-year-old female, died day 28 post-TAVI, cause of death: wound infection, sepsis, multiorgan failure (BNP 2170 baseline vs 1092 $24 \mathrm{~h})$ ). Only the second of these cases died from a cardiac cause. This patient had severe LV impairment before TAVI with EF of $15 \%$. Six hours post-TAVI we observed significant increase in $\mathrm{E} / \mathrm{E}$ ' (11 to 28 ), decrease of $\mathrm{dp} / \mathrm{dt} / \mathrm{EDV}$ (2.7 to $2.2 \mathrm{~mm} \mathrm{Hg} / \mathrm{s} / \mathrm{ml}$ ) and decrease in MPI (0.8 to 0.6).

VARC combined safety end-point at 30 days by was $12 \%(n=5)$.

\section{Haemodynamic measurements}

Changes in haemodynamics are summarised in table 2. TAVI resulted in an immediate increase in $\mathrm{CO}$, systemic vascular resistance, systolic blood pressure and mean arterial pressure. There was no change in diastolic blood pressure. There was a significant increase in heart rate at $24 \mathrm{~h}$ after the procedure.

\section{Echocardiography}

Six hours post-TAVI there was a significant decrease in systolic function as measured by $\mathrm{dP} / \mathrm{dt}_{\max } / \mathrm{EDV}$ (in 27 patients only as no mitral regurgitation was detectable in 15 patients after the procedure), coexistent impairment of diastolic function as indicated by mean E/e' index (figure 1A,B), which was associated with a deterioration in MPI (figure 1C), and an appropriate increase in 3D-derived LA volume (figure 1D). Following this, there was recovery of both systolic and diastolic indices at $24 \mathrm{~h}$. A marker of systolic function, S' increased after $24 \mathrm{~h}$. Concurrent with this recovery, we observed a significant decrease in ESV but not EDV. There were no new wall motion abnormalities noted after TAVI in any case. We observed an increase in E velocities and decrease in ejection at 6 and $24 \mathrm{~h}$ after TAVI.

There was no difference in echocardiographic parameters between patients with and without known ischaemic heart disease.

\section{Markers of myocardial injury}

The above changes in haemodynamics were associated with a significant increase in troponin I levels $(0.07 \pm 0.01$ vs $1.59 \pm 0.21 \mu \mathrm{g} / \mathrm{l}, \quad \mathrm{p}<0.005)$ and $\mathrm{CK}-\mathrm{MB} \quad(1.99 \pm 0.19$ vs $6.82 \pm 0.7 \mathrm{ng} / \mathrm{ml}, \mathrm{p}<0.005) 4-6 \mathrm{~h}$ after the procedure (figure $2 \mathrm{~A}$, $B)$. There was no difference in amount of myocardial injury (assessed by area under the curve for CK-MB) between patients with coexisting coronary artery disease and patients with unobstructed coronary arteries $(7.5 \pm 0.8$ vs $11.0 \pm 1.9$, respectively, NS) or between patients with reduced $(\mathrm{EF}<45 \%)$ and normal LV function (10.1 \pm 1.5 vs 7.6 \pm 1.1 , respectively, NS).

\section{Markers of myocardial dysfunction and neurohormonal activation}

We observed a significant increase of ST2 (30183 vs 38414 pg/ $\mathrm{ml}, \mathrm{p}<0.05$ ), aldosterone (159 vs $403 \mathrm{pg} / \mathrm{ml}, \mathrm{p}<0.05$ ) but not NT-BNP (5498 vs 5385 pg/ml, NS) 4-6 h after TAVI (figure 2C, D). Despite the lack of increase in NT-BNP levels in the group as a whole, raised levels were noted in $30 \%$ of patients after successful TAVI. In addition, there was a significant correlation between myocardial injury (assessed by area under the curve for CK-MB) and changes in NT-BNP $(r=0.34, p<0.0005)$, aldosterone levels $(r=0.56, p<0.0001)$ and ST2 $(r=0.21, p<0.05)$.

\section{DISCUSSION}

This study is the first to describe the occurrence of transient myocardial dysfunction after successful TF-TAVI. This was associated with an increase in the levels of markers of myocardial dysfunction, neurohormonal activation (ST2 and aldosterone) and the release of markers of myocardial injury (troponin I and $\mathrm{CK} M B$ ). This does not appear to be related to the extent of coronary artery disease although the numbers are small. We suggest that this transient postprocedural myocardial dysfunction may be due to myocardial stunning/injury possibly due to subendocardial ischaemia incurred at the time of rapid pacing.

We observed that the release of chronic pressure overload by successful implantation of an aortic valve prosthesis during TAVI results in an immediate increase in CO. In contrast to this, after conventional cardiac surgery patients with diastolic LV

Table 2 Changes in haemodynamic parameters

\begin{tabular}{|c|c|c|c|c|}
\hline & Baseline & $4-6 \mathrm{~h}$ & $2-26 \mathrm{~h}$ & p Value \\
\hline Heart rate (bpm) & $67 \pm 2$ & $71 \pm 2^{*}$ & $78 \pm 2^{*} \dagger$ & $<0.05$ \\
\hline SBP $(m m \quad H g)$ & $108 \pm 3$ & $135 \pm 4^{*}$ & $132 \pm 3^{*} \dagger$ & $<0.05$ \\
\hline $\mathrm{DBP}(\mathrm{mm} \mathrm{Hg})$ & $55 \pm 2$ & $51 \pm 1$ & $52 \pm 2$ & NS \\
\hline MAP (mm Hg) & $73 \pm 2$ & $79 \pm 2^{*}$ & $79 \pm 2^{*}$ & $<0.05$ \\
\hline $\mathrm{CO} \neq(\mathrm{I} / \mathrm{min})$ & $3.7 \pm 0.82$ & $4.5 \pm 1.5^{*}$ & $4.5 \pm 1.3^{*}$ & $<0.05$ \\
\hline SVR $\neq($ dyn.s/cm5) & $1266 \pm 63$ & $1415 \pm 89^{*}$ & $1342 \pm 94^{*}$ & $<0.05$ \\
\hline$S V \neq(\mathrm{ml})$ & $59 \pm 17$ & $62 \pm 4$ & $61 \pm 4$ & NS \\
\hline PRSW‡ (J/ml) & $45 \pm 3$ & $52 \pm 4^{*}$ & $50 \pm 3^{*}$ & $<0.05$ \\
\hline
\end{tabular}

ANOVA followed by Newman-Keuls post hoc test; ${ }^{*} p<0.05$ vs baseline, $\uparrow p<0.05$ vs $6 \mathrm{~h}$ ‡Invasive data for 38 patients.

CO, cardiac output; DBP, diastolic blood pressure; SBP, systolic blood pressure; SV, Stroke volume; SVR, Systemic vascular resistance; MAP, Mean arterial pressure. 
Figure 1 Changes in echocardiographic indices of systolic and diastolic function peri-transcatheter aortic valve implantation. $A$. Changes in $\mathrm{dP} / \mathrm{dt}_{\max } /$ EDV. B. Changes in the index $\mathrm{E} / \mathrm{e}^{\prime}$. C. Changes in myocardial performance index (MPI). D. Changes in LA volume (LAV) ANOVA followed by Newman-Keuls post hoc test for MPI and LAV Friedman test followed by Dunns post hoc test for $\mathrm{dP} / \mathrm{dt}_{\text {max }} / \mathrm{EDV}$ and index $\mathrm{E} / \mathrm{e}^{\prime}$. *p Value $<0.05$ versus baseline (dPdtmax/EDV from 27 patients, as no detectable mitral regurgitation) EDV, End-diastolic volume; LA, Left atrium.
A

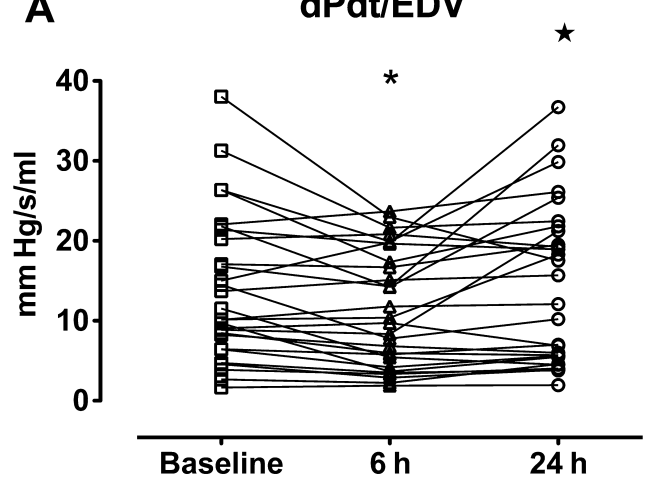

C
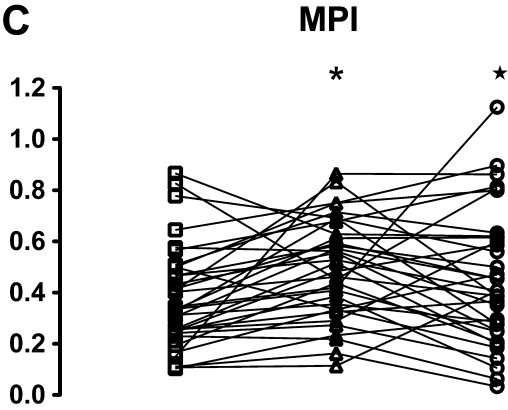

B

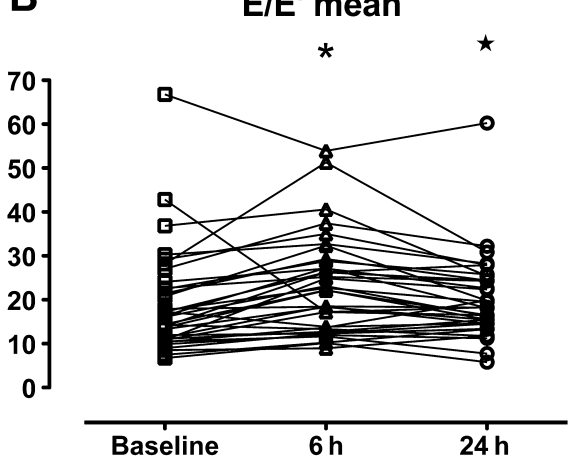

D

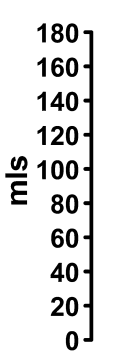

LA Volume

*

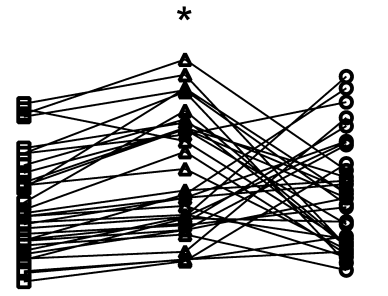

Baseline dysfunction and preserved systolic function have a significant decrease in $\mathrm{CO}^{8}$

Our study provides evidence that in the first $6 \mathrm{~h}$ after successful TF-TAVI there is transient systolic and diastolic dysfunction. These data are based on well validated echocardiographic indices of myocardial performance such as the ratio of early transmitral flow velocity to early-diastolic velocity of the mitral annulus (E/E'), $\mathrm{dP}_{\max } / \mathrm{dt}$ and the MPI. E/E' is an indicator of the diastolic function that correlates well with LV filling pressure. ${ }^{9} \mathrm{dP} / \mathrm{dt}_{\max }$ Is an index characterising $\mathrm{LV}$ systolic function. MPI or Tei index has also been reported to be clinically useful in assessing LV systolic and diastolic function and correlates well with invasive measurements of ventricular function. ${ }^{10}$

The observed perioperative LV impairment is only transient as both TAVI and sAVR have been previously shown to result in improvement of LV function at later time points postprocedure. ${ }^{1}{ }^{11}$ However, the transient disturbances in LV performance we describe are likely to have an impact on early postoperative recovery and may inform appropriate medical management in the immediate postprocedural period. ${ }^{2}$

An additional factor that may contribute to this transient myocardial dysfunction is the sudden release of chronic afterload. It has been previously shown in invasive studies in humans that acute afterload release, for example, by using aortic counterpulsation, results in decrease of $\mathrm{dPdt}$ and end-systolic volumes. ${ }^{12}$ The increase in systemic vascular resistance seen at 6 and $24 \mathrm{~h}$ may occur as a result of this decrease of afterload, but this phenomenon requires further study.

LVH is one of the major compensatory mechanisms to chronic pressure overload. The normal endocardial to epicardial ratio of coronary blood flow is significantly reduced and even reversed in $\mathrm{LVH}$, especially during rapid heart rates. ${ }^{13}$ Bache and Cobb showed that there is rate-dependent decrease in subendocardial blood flow, which decreases myocardial blood flow. Active coronary vasomotion is necessary for the maintenance of uniform transmural perfusion during tachycardia ${ }^{14}$ but it is well known that coronary endothelial function is significantly impaired in patients with LVH and aortic stenosis. ${ }^{15}$ Tarumi et al demonstrated that pacing-induced tachycardia further exacerbates LV diastolic dysfunction in the hypertrophic ventricle, without an immediate effect on global or regional LV systolic function. ${ }^{16}$ All of the patients included in our study had longstanding aortic stenosis with $\mathrm{LVH}$, which made them prone to pacing-related ischaemia during aortic balloon valvuloplasty and Sapien valve deployment.

Previous coronary artery bypass grafting increases predicted operative risk for conventional valve replacement and the presence of coronary artery disease has been demonstrated to increase procedural risk with sAVR. Dewey et al showed that coexisting coronary artery disease negatively impacts procedural outcomes and long-term survival in patients undergoing TAVI. ${ }^{17}$ However, a separate study suggested that the presence of coronary artery disease or non-revascularised myocardium was not associated with an increased risk of adverse events after the procedure. ${ }^{18}$ In our group, $66 \%$ of patients had at least one angiographically significant stenosis ( $>50 \%$ luminal diameter) in a major epicardial coronary artery. There did not appear to be a major difference in myocardial injury according to the presence or absence of coronary disease but patient numbers are too small to analyse in detail.

Myocardial damage during TAVI could be caused by periprocedural conditions, resulting in myocardial oxygen supply-demand mismatch, such as balloon valvuloplasty, acute aortic regurgitation and temporary hypotension during rapid ventricular pacing or even local damage to the subaortic myocardium by the expanded prosthesis. Another potential mechanism for myocardial injury during TAVI is embolisation 
Figure 2 Changes in markers of myocardial injury and neurohormonal activation peri-transcatheter aortic valve implantation (Panel A: CK-MB, Panel B: troponin I, Panel C: ST2 and Panel D: aldosterone). Friedman test followed by Dunns post hoc test (NS, not significant; ${ }^{*} \mathrm{p}<0.05$ vs baseline; $\star \mathrm{p}<0.05$ vs $6 \mathrm{~h}$ ).
A
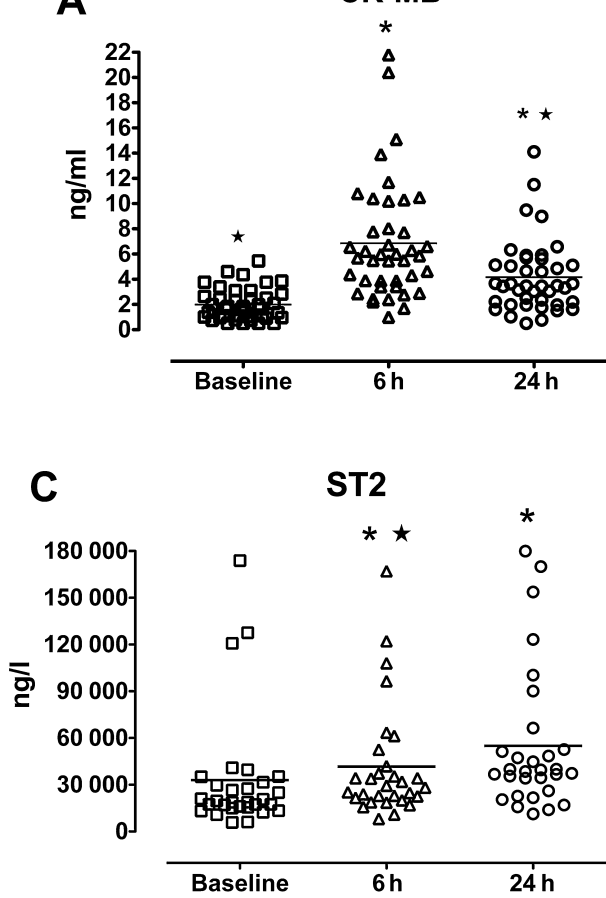
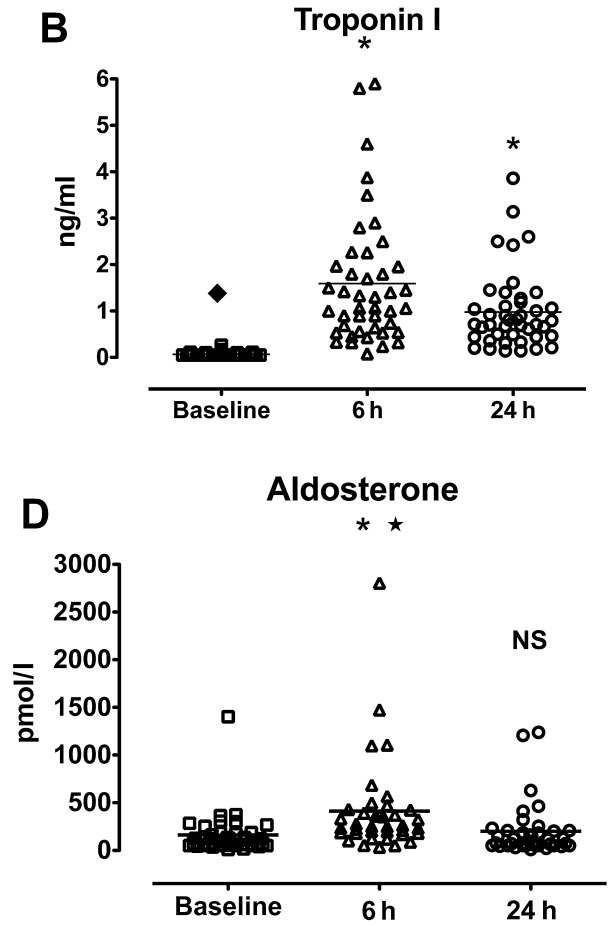

into the coronary arteries. Cardiac magnetic resonance would be a useful way to identify areas of microinfarction and to assess myocardial perfusion before and after TAVI. Moreover, this technique could give further clues as to the mechanism of myocardial damage: global versus regional ischaemia.

ST2 is a member of the interleukin-1 receptor superfamily that was identified as a potential novel biomarker in patients with advanced heart failure. ${ }^{19}$ ST2 is significantly upregulated in acute heart failure ${ }^{20}$ and it correlates well with early postinfarction LV dysfunction. ${ }^{21}$ In contrast to NT-pro-BNP protein ST2 levels are not associated with clinical futures leading to chronic increase of LV wall stress, which is why it may be a better marker of acute heart failure. ${ }^{22}$ We have shown for the first time that there is a significant increase in ST2 levels after successful TAVI, perhaps a response to periprocedural myocardial dysfunction. The clinical significance of this finding requires further investigation.

Similar to ST2, aldosterone has recently been identified as a predictor of mortality in chronic heart failure. ${ }^{23}$ As in acute heart failure syndromes, we observed a significant increase in aldosterone levels immediately after TAVI. Aldosterone rise in the short-term causes electrolyte imbalance, water retention and microvascular dysfunction and in the long-term could affect cardiac remodelling. ${ }^{24}$

BNP secretion after cardiac surgery has been attributed to myocardial infarction and LV dysfunction due to myocardial stretch. ${ }^{25}$ This BNP stimulation by surgical trauma still occurs in the absence of cardiopulmonary bypass, as reported in off-pump coronary artery surgery procedures in which the systematic stress hormone response was comparable with conventional surgical revascularisation. ${ }^{26}$ In addition, perioperative BNP levels have a well documented prognostic importance in cardiac surgery. ${ }^{27}$ Postoperative elevated BNP concentrations have been associated with prolonged hospital stay and postoperative cardiac dysfunction. ${ }^{2}$ In our study, we have not observed a significant increase in BNP, which may be because the cardiac dysfunction was relatively transient and mild. However, BNP was increased over $10 \%$ compared with baseline in $30 \%$ of patients.

These findings have an implication on immediate postprocedural care. Patients after TAVI become tachycardic on the first day postprocedure and quite often develop peripheral oedema. We postulate that this postprocedural tachycardia is a response to observed transient myocardial dysfunction. Knowledge of LV contractility and neurohormonal activation may help in choosing appropriate inotropic agents and aid in decision-making regarding the introduction of diuretics, $\beta$ blockers and ACEIs.

\section{CONCLUSIONS}

These data demonstrate for the first time that within the first $24 \mathrm{~h}$ of a successful TF-TAVI, both systolic and diastolic dysfunction occurs. The abrupt release of chronic pressure overload results in an overall increase in $\mathrm{CO}$ despite this depression in myocardial performance. These phenomena are associated with an increase in serum markers of myocardial injury and dysfunction. The rise in the markers of myocardial injury suggests that the postprocedural dysfunction may be due to myocardial stunning and periprocedural myocardial damage. However, recovery of contractility is observed very quickly after TF-TAVI and the long-term effects of observed transient LV dysfunction are unknown. These observations help our understanding of haemodynamics in the immediate post-TAVI phase and will inform appropriate management of the recovering patient in the intensive care unit. The long-term effect of TAVI on myocardial performance is the subject of ongoing studies.

Acknowledgements $\mathrm{RD}$ is a visiting Senior Lecturer from Department of Cardiology, Medical University of Gdansk, Poland.

Contributors RD, OW, MJM, JB, EA, RK, AMS and PAM took part in designing the study and interpretation of all data. RD and PAM prepared the manuscript. RD, AB, LS and PP analysed echocardiographic data. RD, RS, TD and BB analysed and performed all biochemical tests. 
Funding This study was supported by the King's College Hospital NHS Foundation Trust R\&D Initiative Grant.

Competing interests None.

Ethics approval Ethics approval was provided by the King's College Hospital Ethics Committee.

Provenance and peer review Not commissioned; externally peer reviewed.

\section{REFERENCES}

1. Guarracino $\mathbf{F}$, Talini $E$, Landoni $G$, et al. Effect of aortic valve surgery on left ventricular diastole assessed by echocardiography and neuroendocrine response: percutaneous versus surgical approach. J Cardiothorac Vasc Anesth 2010;24:25-9.

2. Provenchère $\mathbf{S}$, Berroeta $\mathrm{C}$, Reynaud $\mathrm{C}$, et al. Plasma brain natriuretic peptide and cardiac troponin I concentrations after adult cardiac surgery: association with postoperative cardiac dysfunction and 1-year mortality. Crit Care Med 2006:34:995-1000.

3. Dworakowski R, MacCarthy PA, Monaghan M, et al. Transcatheter aortic valve implantation for severe aortic stenosis-a new paradigm for multidisciplinary intervention: a prospective cohort study. Am Heart J 2010:160:237-43.

4. Leon MB, Piazza N, Nikolsky E, et al. Standardized endpoint definitions for Transcatheter Aortic Valve implantation clinical trials: a consensus report from the Valve Academic Research Consortium. J Am Coll Cardiol 2011;57:253-69.

5. Bhan A, Kapetanakis S, Monaghan MJ. Three-dimensional echocardiography. Heart 2010;96:153-63.

6. Kawai H, Yokota Y, Yokoyama M. Noninvasive evaluation of contractile state by left ventricular $\mathrm{dP} / \mathrm{dtmax}$ divided by end-diastolic volume using continuous-wave Doppler and M-mode echocardiography. Clin Cardiol 1994:17:662-8.

7. Tei C, Nishimura RA, Seward JB, et al. Noninvasive Doppler-derived myocardial performance index: correlation with simultaneous measurements of cardiac catheterization measurements. J Am Soc Echocardiogr 1997:10:169-78.

8. Shim JK, Choi YS, Chun DH, et al. Relationship between echocardiographic index of ventricular filling pressure and intraoperative haemodynamic changes during offpump coronary bypass surgery. Br J Anaesth 2009;102:316-21.

9. Ommen SR, Nishimura RA, Appleton CP, et al. Clinical utility of Doppler echocardiography and tissue Doppler imaging in the estimation of left ventricular filling pressures: a comparative simultaneous Doppler-catheterization study. Circulation 2000;102:1788-94.

10. LaCorte JC, Cabreriza SE, Rabkin DG, et al. Correlation of the Tei index with invasive measurements of ventricular function in a porcine model. J Am Soc Echocardiogr 2003; 16:442-7.

11. Bauer $\mathbf{F}$, Bénigno $S$, Lemercier $M$, et al. Early improvement of left ventricula function after implantation of a transcutaneous aortic valve: a tissue Doppler ultrasound study. Arch cardiovascular diseases 2009:102:311-18.
12. Schreuder JJ, Maisano F, Donelli A, et al. Beat-to-beat effects of intraaortic balloon pump timing on left ventricular performance in patients with low ejection fraction. Ann Thorac Surg 2005;79:872-80.

13. Marcus ML, Doty DB, Hiratzka LF, et al. Decreased coronary reserve: a mechanism for angina pectoris in patients with aortic stenosis and normal coronary arteries. N Engl J Med 1982;307:1362-6.

14. Bache RJ, Cobb FR. Effect of maximal coronary vasodilation on transmural myocardial perfusion during tachycardia in the awake dog. Circ Res 1977:41:648-53

15. Bozbas H, Pirat B, Yildirir A, et al. Coronary flow reserve is impaired in patients with aortic valve calcification. Atherosclerosis 2008;197:846-52.

16. Tarumi T, Takebayashi S, Fujita $\mathrm{M}$, et al. Pacing tachycardia exaggerates left ventricular diastolic dysfunction but not systolic function and regional asynergy or asynchrony in patients with hypertrophic cardiomyopathy. Europace 2010;12:1308-15.

17. Dewey TM, Brown DL, Herbert MA, et al. Effect of concomitant coronary artery disease on procedural and late outcomes of transcatheter aortic valve implantation. Ann Thorac Surg 2010;89:758-67; discussion 767

18. Masson JB, Lee M, Boone RH, et al. Impact of coronary artery disease on outcomes after transcatheter aortic valve implantation. Catheter Cardiovasc Interv 2010;76:165-73

19. Weinberg EO, Shimpo M, Hurwitz $S$, et al. Identification of serum soluble ST2 receptor as a novel heart failure biomarker. Circulation 2003:107:721-6.

20. Januzzi JL, Peacock WF, Maisel AS, et al. Measurement of the interleukin family member ST2 in patients with acute dyspnea: results from the PRIDE (Pro-Brain Natriuretic Peptide Investigation of Dyspnea in the Emergency Department) study. J Am Coll Cardiol 2007; 50:607-13.

21. Weir RA, Miller AM, Murphy GE, et al. Serum soluble ST2: a potential novel mediator in left ventricular and infarct remodeling after acute myocardial infarction. J Am Coll Cardiol 2010;55:243-50

22. Sabatine MS, Morrow DA, Higgins LJ, et al. Complementary roles for biomarkers of biomechanical strain ST2 and N-terminal prohormone B-type natriuretic peptide in patients with ST-elevation myocardial infarction. Circulation 2008;117:1936-44.

23. Guder G, Bauersachs J, Frantz S, et al. Complementary and incremental mortality risk prediction by cortisol and aldosterone in chronic heart failure. Circulation 2007:115:1754-61.

24. Albaghdadi M, Gheorghiade M, Pitt B. Mineralocorticoid receptor antagonism: therapeutic potential in acute heart failure syndromes. Eur Heart $J$ 2011:32:2626-33.

25. Sinha AM, Breithardt OA, Schmid M, et al. Brain natriuretic peptide release in cardiac surgery patients. Thorac Cardiovasc Surg 2005;53:138-43.

26. Masuda M, Morita S, Tomita $\mathrm{H}$, et al. Off-pump CABG attenuates myocardial enzyme leakage but not postoperative brain natriuretic peptide secretion. Ann Thorac Cardiovasc Surg 2002;8:139-44.

27. Cuthbertson BH, Croal BL, Rae D, et al. N-terminal pro-B-type natriuretic peptide levels and early outcome after cardiac surgery: a prospective cohort study. $\mathrm{Br} \mathrm{J}$ Anaesth 2009:103:647-53. 